\title{
STEREO EVALUATION OF ALOS PRISM AND IKONOS IN YEMEN
}

\author{
Rupert Müller ${ }^{1}$, Mathias Schneider ${ }^{1}$, P. V. Radhadevi ${ }^{2}$, Peter Reinartz ${ }^{1}$, Friedhelm Schwonke ${ }^{3}$ \\ ${ }^{1)}$ German Aerospace Center (DLR) \\ Remote Sensing Technology Institute, 82234 Wessling, Germany \\ e-mail: \{Rupert.Mueller, Mathias.Schneider, Peter.Reinartz\}@dlr.de \\ ${ }^{2)}$ ADRIN (Advanced Data processing Research INstitute), \\ Department of Space, Manovikasnagar P.O., Secunderabad 500 009, India \\ e-mail: drpvr@adrin.res.in \\ ${ }^{3)}$ Federal Institute for Geosiences and Natural Resources (BGR), 30655 Hannover, Germany \\ e-mail: friedhelm.schwonke@bgr.de
}

\begin{abstract}
DLR's Remote Sensing Technology Institute has a long lasting experience in developing spaceborne stereo scanners (MEOSS, MOMS) and the corresponding photogrammetric software systems for stereo evaluation and orthorectification. It takes part in the ESA/JAXA-AO Program to evaluate the performance and potential of the three-line stereo scanner PRISM and the multispectral imaging sensor AVNIR-2 on-board the Japanese satellite ALOS as a principal investigator. The high geometric resolution of PRISM (2.5 $\mathrm{m}$ ground sampling distance at nadir) combined with the medium swath width of $35 \mathrm{~km}$ has the potential to achieve high quality Digital Elevation Models up to 1:25.000 scale topographic maps for various applications. One of the proposed test sites is located near Sana'a, Yemen, where additionally to the PRISM stereo data also an IKONOS stereo image pair exists, which is used for DEM comparison and performance analysis. The results of this test site are evaluated in cooperation with the Advanced Data processing Research INstitute (ADRIN), India and the Federal Institute for Geosciences and Natural Resources (BGR), Hannover.
\end{abstract}

\section{Index Terms - DEM, ALOS PRISM, IKONOS}

\section{INTRODUCTION}

The paper gives a report on the generation of digital elevation models (DEM) from ALOS/PRISM 3-line stereo images using three different methodologies. The results are compared to a DSM derived from IKONOS stereo images, which serves as reference.

The physical model of the pushbroom satellite imaging process relates a point in an earth-bound object coordinate system to the position of its projection in an image file coordinate system. In this paper the results from different sensor models are compared. The first one is a specific sensor model designed for ALOS, incorporating the precise payload geometry, corrections for the relative alignment of the CCD chips, corrections for light aberration and atmospheric refraction. For this model a set of improved correction parameters provided by JAXA are used [1] [3][12]. The second one is a generic sensor model with self calibration [7][8][9]. Interior orientation parameters are also included into the adjustment and no information of the payload geometry, describing the position of the individual CCDs, is used. The third one uses Rational Polynomial Functions RPF, which actually is derived from the specific sensor model.

Ground control points (GCP) of sufficient accuracy are introduced to increase the geometric accuracy of the derived DSM, but as well as evaluations without using GCP demonstrate the pointing performance of the ALOS/PRISM system.

The possible potential using high quality DEMs is illustrated for an integrated water resource management in Amran (Yemen).

\section{DATA BASIS}

For the area around the city Shibam (north-west of Sana) in Yemen the following data sets for the investigations are used:

1) Ikonos stereo images from $09^{\text {th }}$ March 2007 with a ground resolution of $1 \mathrm{~m}$.

2) PRISM triple stereo image from $24^{\text {th }}$ March 2008 with a ground resolution of $2.5 \mathrm{~m}$.

\section{DEM GENERATION}

In a first step of the DEM generation process (PRISM and IKONOS stereo scenes) tie points between the stereo image partners are identified using a hierarchical intensity based matching and densified by a region growing algorithm. Finally the object space coordinates are reconstructed by forward intersection based on modeling of the imaging process (further on called Direct Models \#1 and \#2) or on Rational Polynomial Functions (further on called RPC model).

\subsection{Image Matching}

Based on the Foerstner interest operator pattern windows are selected in one of the images and located with an accuracy of about one pixel in the other image via the maximum of the normalized correlation coefficients computed by sliding the pattern area all over the search area. The search areas in the matching partner image are determined by estimation of local affine transformations based on already available tie points in the 
neighborhood (normally from a coarser level of the image pyramid). The approximate tie point coordinates are then refined to sub-pixel accuracy by local least squares matching and in a further step densified by region growing using the concept of Otto and Chau. It combines LSM with a strategy for local propagation of initial conditions of LSM. Various methods for blunder reduction are applied for both steps of the matching:

- Threshold for correlation coefficient

- 2-directional matching and threshold on resulting shifts of the coordinates

- Threshold on residuals (in image space) from forward intersection based on the rigorous modeling of the imaging process or on Rational Polynomial Functions.

The matching process finally provides mass points used for comparison of different techniques of object point reconstruction (see following chapters) and for interpolation to a regular grid.

\section{PRISM PROCESSING}

One of the optical instruments on the Japanese satellite ALOS is the three line stereo sensor PRISM having a forward, backward and nadir view panchromatic channels $(0.52-0.77 \mu \mathrm{m})$ with a ground sampling distance of about $2.5 \mathrm{~m}$ at nadir and a stereo angle of $23.8^{\circ}$ between forward and backward direction according to a base to height ratio of one. The focal plane arrays consist of 8 CCD lines for the forward and backward view and 6 CCD lines for the nadir view, which build a line of 14496 pixels corresponding to a swath with of about $35 \mathrm{~km}$ (only up to 4 CCD arrays are active during image acquisition). The JAXA standard data product type Level 1B1 serves as input for the investigations, which are corrected for systematic and radiometric effects resulting in Topof-Atmosphere radiance values. Because the lossy on board JPEG compression using variable quantization leads to image artifacts, which are minimized by a 3x3 Gaussian filter in order to increase image matching performance quality and to reduce matching outliers.

\subsection{Ground Control Point Determination}

In-situ ground control point (GCP) measurements are not available for the Yemini test site. Therefore the geocoded IKONOS scene (using the RPCs) together with bilinear interpolated terrain height information from DEMs derived from SRTM data serve as source for GCP determination. For each of the three stereo partners 6 GCPs are manually measured. Unfortunately the IKONOS image is falling only in the first half of the PRISM image.

\subsection{Direct Model (\#1)}

The specific sensor model designed for PRISM includes the orbit and attitude product as well as geometric correction tables, which are extracted from the ancillary files delivered in CEOS format. The very sophisticated sensor model of the PRISM images include the following transformations from pixel physical numbering to earth bound coordinates [1][3][12].

- from pixel absolute column number to pixel look direction angles using pre- and post-launch geometric calibration values

- from pixel look direction vectors described in the sensor coordinate frame to the body coordinate frame defined by the Star Tracker System (STS). This transformation includes the initial alignment angles measured in laboratory, the long term behavior of the mounting angles since satellite launch (including gravity release effects) as well as short term variation due to thermal distortions as a function of the satellite sun exposure time during orbit revolution.

- from body coordinate frame to earth centered inertial ECI frame using unit quaternions measured with $10 \mathrm{~Hz}$ synchronized to the image line acquisition time by Lagrange interpolation (the quaternions are transformed to Euler angles for further processing).

- from the ECI frame to the earth centered ECR frame using the nutation / precession matrix, the polar motion matrix and the earth rotation

- the position coordinates of the sensor projection center are derived from GPS measurements reduced by the pre-launch calibrated lever arm values

- Additionally the atmospheric refraction as well as the light aberration [Greslou 2008] is taken into account

The GCPs are used to estimate for each of the three stereo images additional rotation angles describing the alignment between the sensor and the body coordinate frame (boresight alignment angles) as described in [6].

\subsection{RPC Model}

From the direct model \#1 -improved by GCP information - a three dimensional grid of object point coordinates ( 25000 virtual points) is generated from where Rational Polynomial Coefficients (RPC) are derived as described in [4][5].

\subsection{Direct Model (\#2)}

A generic sensor model for georeferencing of linear CCD array images has been developed at ADRIN. This model is very flexible and has been successfully used for the orientation of SPOT-1, IRS1C/1D, TES, IRS-P6 and IRS-P5 [7][8][9] The algorithm is based on the viewing geometry of the satellite, combining the principles of photogrammetric collinearity equations. The functionality to accommodate ALOS/PRISM imagery has been added to this model. Merged level 1B1 images are used. We use a self calibration approach in which interior orientation parameters are also included in the adjustment. Information on the payload geometry provided by JAXA is not used in the model. Nominal pre-launch camera focal length is used as initial approximation. Boresight alignment angles are initialized to zero. Satellite ephemeris, attitude, image start time and number of dummy pixels on the first and last detector arrays are used. With these values, initial fitting of the trajectory is done. A generic polynomial model is developed so that by selecting the order of polynomial, it can be adapted for different types of sensors. For example, the order of the polynomial model which is up-linked will be higher for agile satellites compared to non-agile satellites. The user can select the parameters and the order up to which he has to correct for the coefficients. If the post calibrated values of the payload parameters are included for a particular sensor, he can exclude the interior orientation parameters from the adjustment. For ALOS, the framelet co-ordinate system is not used. Instead, the local coordinates of the combined CCD array (virtual) are used and centre of this is used as the geometric origin for the image co-ordinates. The mounting of this point is initialized to zero and computed through the GCPs. 


\section{DEM COMPARISONS}

The DEMs derived from the PRISM stereo images are compared to the DEM derived from IKONOS stereo images, which further serves as reference.

Figure 1 shows a profile between the IKONOS DEM and the PRISM DEMs that are generated with the Direct Model \#1 using improved parameters by GCPs and without using GCP information.

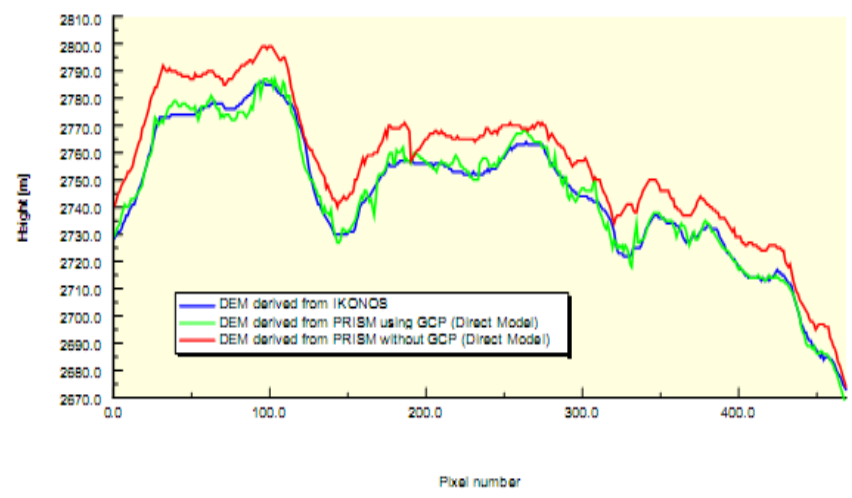

Figure 1 Profiles of the IKONOS reference DEM (blue), the PRISM DEMs generated without using GCP information (red) and using GCPs for sensor model improvements (green).

The DEM profiles show a good lateral correlation. The shift values between IKONOS and PRSIM DEM (with GCP used) are $3.5 \mathrm{~m}$ (east), $-1.5 \mathrm{~m}$ (north) and $0.31 \mathrm{~m}$ (height). But also using no GCP for the parameter improvements (only the in-flight measured exterior orientation and the geometric calibration parameters) the DEM shift values are $14.1 \mathrm{~m}$ (east), $-12.0 \mathrm{~m}$ (north) and $-11.7 \mathrm{~m}$ height, which shows the high geometric performance of ALOS PRISM images, also reported in [10].

Comparing the profiles between the DEMs generated with the different forward intersection techniques (see figure 2) a slight difference between the direct models and the RPC model is observable, whereas the results produced by the two direct models are very similar (figure 3).

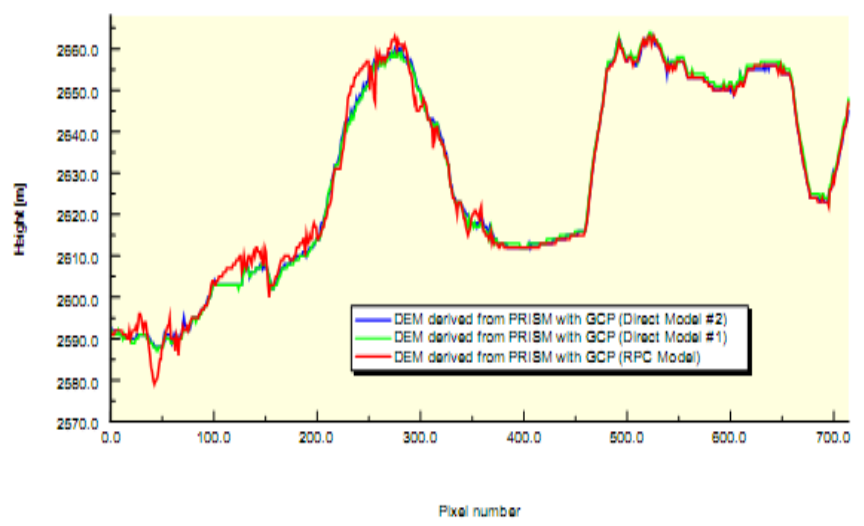

Figure 2 Profiles of the PRISM DEMs generated by forward intersection using the direct model \#1 (green), the direct model \#2 (blue) and the RPC model using in all cases the same GCP information.

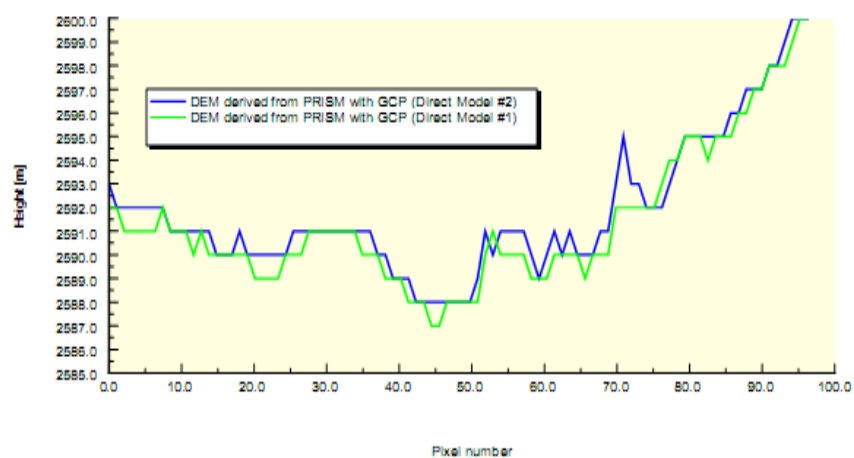

Figure 3 Detailed profiles of the PRISM DEMs generated by forward intersection using the direct model \#1 (green), the direct model \#2 (blue). The mean height difference is lower than one meter.

The 3D deviations and shifts at the mass points between the different models are given in table 1 .

\begin{tabular}{|c|c|c|c|c|}
\hline & Mean [m] & Sigma $[\mathrm{m}]$ & RMSE [m] & Model \\
\hline $\mathrm{y}$ & -3.04 & 0.43 & 3.07 & \multirow{3}{*}{$\begin{array}{l}\text { Direct Model \#1 } \\
\text { vs. RPC Model }\end{array}$} \\
\hline $\mathrm{x}$ & 2.79 & 0.36 & 2.81 & \\
\hline $\mathrm{z}$ & -0.68 & 1.17 & 1.35 & \\
\hline $\mathrm{y}$ & -5.24 & 110 & 5.35 & \multirow{3}{*}{$\begin{array}{l}\text { Direct Model \#2 } \\
\text { vs. RPC Model }\end{array}$} \\
\hline $\mathrm{x}$ & -0.51 & 2.03 & 2.09 & \\
\hline $\mathrm{z}$ & -0.34 & 0.36 & 0.50 & \\
\hline
\end{tabular}

Table 1 Comparison between RPC Model and the two Direct Models \#1 and \#2 using the same mass points of about $2 \cdot 10^{6}$ values.

Comparing the object points (produced by the direct model) of the 3D artificial grid used for RPC calculation with the re-calculated values using the RPCs slight residuals can be observed, which is shown in the plots of figure 4 and figure 5 .

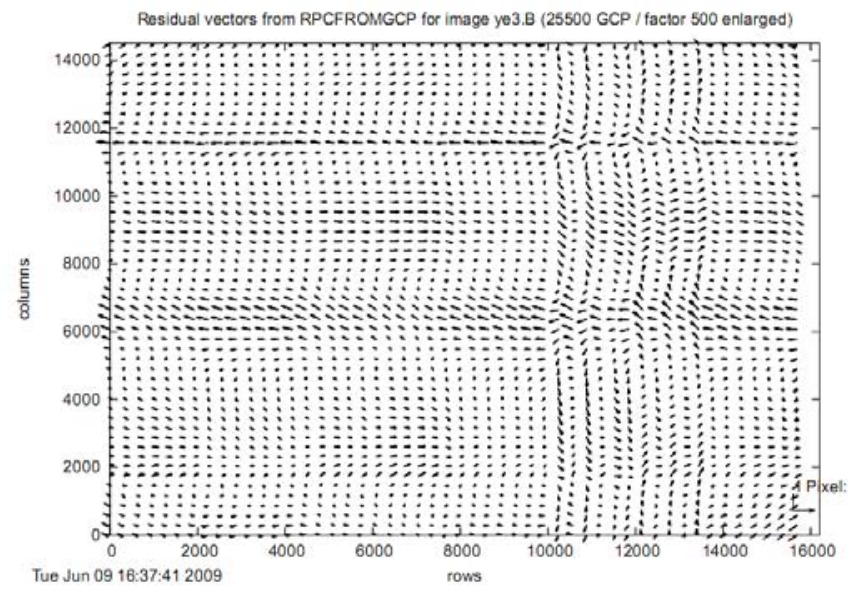

Figure 4 Residual plot between object points coordinates derived from the direct model and those re-calculated by RPCs for backward image of the PRISM sensor. At bottom-right the size of one pixel is shown. 


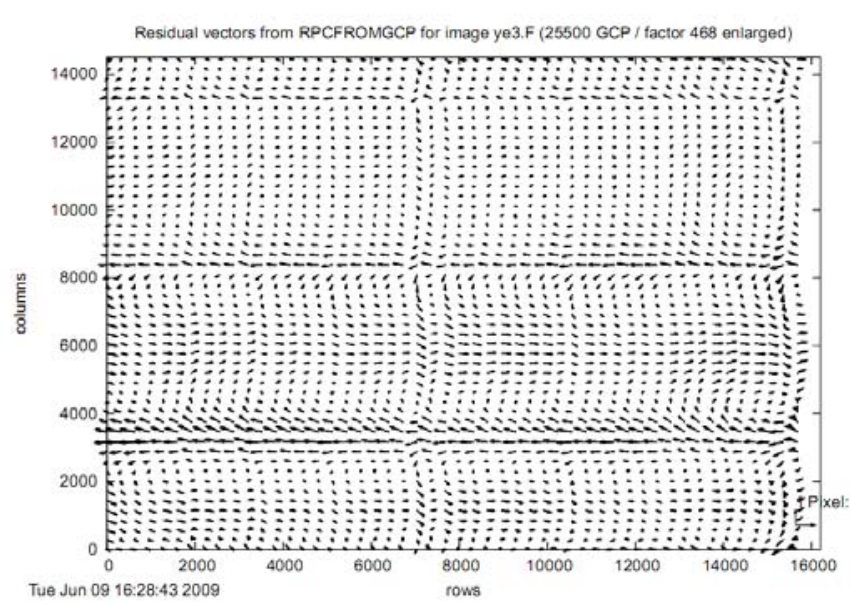

Figure 5 Residual plot between object points coordinates derived from the direct model and those re-calculated by RPCs for the forward image of the PRISM sensor. At bottom-right the size of one pixel is shown.

The residuals are smaller than one pixel size (superimposed it can be greater than one pixel size) and seem to affect the DEM generation using RPC especially for this high resolution imagery. In column direction the individual CCD arrays building the image line and in row direction an oscillation, possible linked to the oscillation of the pitch angle caused by the satellite reaction wheels, are visible.

\section{APPLICATIONS}

Within the ALOS ADEN AO Project ID 3551 two possible applications in Yemen are proposed illustrating the potential of the use of high quality DEMs.

The availability of water (drinking water and irrigation of agrarian areas) is of increasing importance in developing countries. High resolution satellite data and derived products with appropriate methods provide important information of the changing agrarian areas and resultant water supplies. Localisation and assessment of potential location for water dams can be derived from high quality DEMs with horizontal resolution better than $5 \mathrm{~m}$ - as valid for the PRISM derived DEMs. This information is an important constituent of a future GIS system established in this area of Yemen. A second example is the terrace cultivation in Yemini mountain valley environment, which is endangered by mass movements, particularly rock and land slides triggered by flash flood after heavy rainfalls.

Susceptibility mapping and monitoring using DEM and land use information from ALOS PRISM (as well as AVNIR-2 multispectral) data can contribute to these topics.

\section{CONCLUSIONS}

From ALOS/PRISM stereo images DEMs are derived applying different methods - namely the specific sensor model for PRISM and a generic sensor model. It was shown that the achieved accuracy for georeferencing through both sensor models is very similar and close to the reference data set. Using RPC slight differences of pixel magnitude to the sensor models are obtained, which means that not all effects (e.g. oscillations during image acquisition, relative alignment of the CCD assembly) can be captured by the RPC model and influence the geometric accuracy of the DEM product. Applying RPC for sensors with very high geometric resolution therefore should be handled carefully.

\section{REFERENCES}

[1] JAXA, ALOS Algorithm description (PRISM/AVNIR-2), 2006

[2] D. Greslou, F. De Lussy, J. Montel, Light Aberration Effect in HR Geometric Model, The International Archives of the Photogrammetry, Remote Sensing and Spatial Information Sciences. Vol. XXXVII. Part B1. Beijing 2008

[3] Kamiya I., Geometric Characteristics of the Early Products of ALOS PRISM,. Bulletin of the Geographical Survey Institute, vol.54, pp.75-82, 2007

[3] Lehner M., Gill, R.S., Semi-automatic derivation of digital elevation models from stereoscopic 3-line scanner data, IAPRS, Vol. 29, Part B4, Washington, USA, pp. 68-75, 1992

[4] Lehner M., Müller R., Reinartz P., DSM and Orthoimages from QuickBird and Ikonos Data Using Rational Polynomial Functions, Proceedings of "High Resolution Earth Imaging for Geospatial Information”, May 17-20, Hannover, Germany, 2005

[5] Lehner M., Müller R., Reinartz P., Schroeder M., Stereo evaluation of Cartosat-1 data for French and Catalonian test sites, Proceedings of the ISPRS Hannover Workshop 2007 High Resolution Earth Imaging for Geospatial Information, Hannover, Germany, May 29 - June 1, 2007

[6] Müller, R., Lehner, M., Reinartz, P., Schroeder, M., Evaluation of Spaceborne and Airborne Line Scanner Images using a generic Ortho Image Processor, Proc. of High Resolution Earth Imaging for Geospatial Information, ISPRS Hannover Workshop, Commision I WG 5, 2005

[7] Radhadevi, P.V., Ramachandran R., Orbit Attitude Modelling of SPOT imagery with a Single Ground Control Point, Photogrammetric Record, 14(84), pp. 973-982, 1994

[8] Radhadevi, P.V., Pass Processing of IRS-1C/1D PAN subscene blocks, ISPRS Journal of Photogrammetry \& Remote Sensing, 54, 5, 1999.

[9] Radhadevi, P.V., S. S. Solanki, S.S., Inflight calibration of multiple camera of IRS-P6 - Photogrammetric Record,23(121), pp 69-89, 2008

[10] Saunier S., Demange C., Goryl, P., Final calibration / validation report PRISM, 2007

[11] Schneider, M., Lehner M., Müller, R., Reinartz, P., Stereo Evaluation of ALOS/PRISM Data on ESA-AO Test Sites - First DLR Results; Proc. of 'ALOS PI 2008 Symposium”, Island of Rhodes, Greece 3-7 November 2008, (ESA SP-664, January 2009)

[12] Tadono T., Shimada M., Watanabe M., Hashimoto T., Iwata T., Calibration and Validation of PRISM Onboard ALOS, International Archives of Photogrammetry, Remote Sensing and Spatial Information Sciences, Vol.XXXV part B1, pp. 13-18, 2004 